\title{
Hydraulic Fracture and Toughening of a Brittle Layer Bonded to a Hydrogel
}

\author{
Alessandro Lucantonio, ${ }^{1}$ Giovanni Noselli, ${ }^{1}$ Xavier Trepat, ${ }^{2,3,4}$ Antonio DeSimone, ${ }^{1, *}$ and Marino Arroyo ${ }^{5, \dagger}$ \\ ${ }^{1}$ SISSA-International School for Advanced Studies, via Bonomea 265, 34136 Trieste, Italy \\ ${ }^{2}$ Institute for Bioengineering of Catalonia (IBEC), Baldiri Reixac 15-21, 08028 Barcelona, Spain \\ ${ }^{3}$ Institució Catalana de Recerca $i$ Estudis Avançats, 08010 Barcelona, Spain \\ ${ }^{4}$ Facultat de Medicina, Universitat de Barcelona, 08036 Barcelona, Spain \\ ${ }^{5}$ Universitat Politècnica de Catalunya-BarcelonaTech, C/ Jordi Girona 1, 08034 Barcelona, Spain
}

(Received 30 April 2015; published 28 October 2015)

\begin{abstract}
Brittle materials propagate opening cracks under tension. When stress increases beyond a critical magnitude, then quasistatic crack propagation becomes unstable. In the presence of several precracks, a brittle material always propagates only the weakest crack, leading to catastrophic failure. Here, we show that all these features of brittle fracture are fundamentally modified when the material susceptible to cracking is bonded to a hydrogel, a common situation in biological tissues. In the presence of the hydrogel, the brittle material can fracture in compression and can hydraulically resist cracking in tension. Furthermore, the poroelastic coupling regularizes the crack dynamics and enhances material toughness by promoting multiple cracking.
\end{abstract}

DOI: 10.1103/PhysRevLett.115.188105

Brittle materials fracture under tensile [1] or shear [2] stress. Fracture propagation can also occur because of a pressurized fluid filling a preexisting cavity in the material [3]. Recently, a counterintuitive new fracture mode has been identified in epithelial tissues, by which a material susceptible to fracture (a cell monolayer) develops opening cracks under compression [4]. This behavior is caused by the poroelastic coupling in the extracellular matrix, a hydrogel supporting the largely impermeable cell monolayer. Compression leads to an increase of solvent pressure within the gel, which in turn opens cracks hydraulically at cell-cell junctions. This phenomenology suggests that the coupling between deformation and fluid pressure in hydrogels could change the fracture behavior of an adjacent material in unexpected ways. Despite that impermeable thin layers adhered to a hydrogel are ubiquitous in biology [5] and technology [6,7], poroelastic fracture physics under stretch has not been examined systematically.

To address this issue, we first develop a mathematical model to understand when and why cracks propagate in a brittle material adhered to a poroelastic substrate, which captures the main physics for short cracks and at short times. This model reveals different scenarios depending on strain sign and material properties. We then validate this model with fully nonlinear finite element simulations for a single and multiple cracks. These simulations highlight how the coupling with the hydrogel induces material

Published by the American Physical Society under the terms of the Creative Commons Attribution 3.0 License. Further distribution of this work must maintain attribution to the author(s) and the published article's title, journal citation, and DOI.
PACS numbers: 87.19.R-, 46.50.+a, 47.56.+r, 87.85.em

toughening and flaw-tolerance, landmark properties of biological materials $[4,8-10]$.

We consider an infinite brittle solid that contains a single edge crack of initial length $a_{0}$ and is bonded to an infinite hydrogel substrate [Fig. 1(a)]. The system is initially stressfree, the solvent pressure in the hydrogel is zero, and the crack is closed and hydraulically connected to the hydrogel. We assume for now that the brittle solid (hydrogel) is a linear elastic (poroelastic) material in plane strain conditions. In agreement with the small-scale biological context that motivates us, we ignore inertial forces.

The system is then subjected to a remote longitudinal time-dependent strain $\varepsilon(t)$. In the following, we omit the explicit dependence on time of the model variables. The far-field stress in the brittle solid is $\sigma_{\infty}=\bar{E} \varepsilon$, with $\bar{E}=E /\left(1-\nu^{2}\right), E$ the Young's modulus, and $\nu$ the Poisson's ratio. The applied strain changes the pressure distribution of the solvent in the hydrogel. Far away from the crack, the solvent pressure can be computed assuming that the medium is undrained (incompressible) as $p_{g}=-2 G \varepsilon$, where $G$ is the shear modulus of the hydrogel [11]. We note that $p_{g}$ is positive under compression and negative under tension. In general, this bulk pressure will differ from that at the crack inlet $p$, inducing a pressure difference $\Delta p=p_{g}-p$ over a length scale $\ell$, which drives solvent exchange between the hydrogel and the crack cavity.

Based on Darcy's law and estimating the pressure gradient near the crack inlet as $\Delta p / \ell$, we approximate the flux of solvent volume (per unit depth) from the hydrogel to the crack cavity as $q=\delta k \Delta p / \ell$, where $\delta$ is the crack inlet opening and $k$ a material constant that controls solvent diffusivity within the hydrogel $[12,13]$. We 


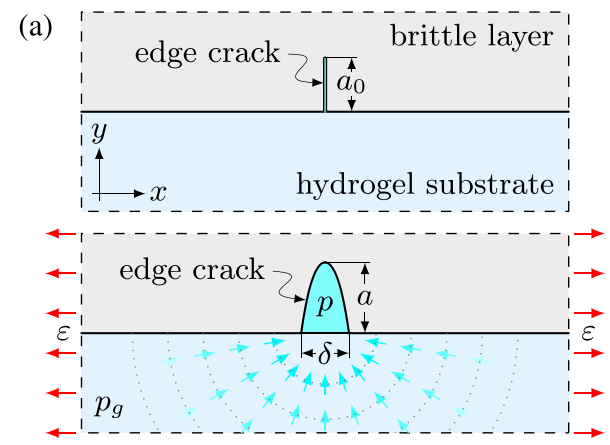

(b)
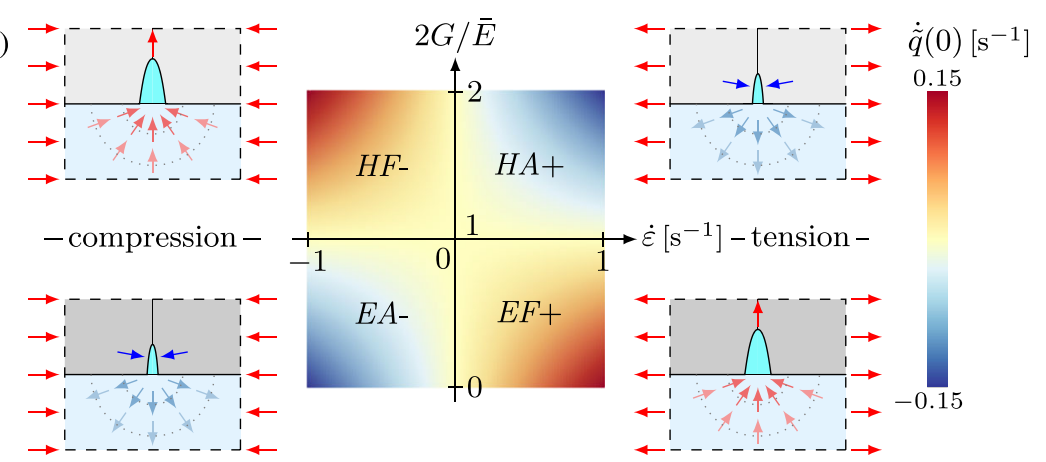

FIG. 1 (color online). Sketch of the model system at rest and under stretch (a). Different crack opening or closing scenarios (b), depending on strain rate $\dot{\varepsilon}$ and on the ratio of the shear modulus $G$ of the hydrogel to the effective Young's modulus $\bar{E}$ of the brittle material. The color map represents the time rate of the solvent flux towards the crack cavity immediately after strain application, $\dot{\tilde{q}}(0)=\dot{q}(0) /(k \bar{E})=\dot{\varepsilon} \zeta / \gamma$. Its sign and magnitude express the tendency and rate of the crack opening or closing. The darker gray shade represents a stiffer brittle layer relative to the hydrogel. The labels in (b) describe the dominant driving force at the crack, hydraulic $(H)$ or elastic $(E)$; the phenomenology, fracture $(F)$ or crack arrest $(A)$; and the nature of the imposed deformation, tensile $(+)$ or compressive $(-)$. For instance, $H A+$ stands for hydraulic $(H)$ arrest $(A)$ under tension $(+)$.

further assume that the length scale $\ell$ is proportional to the crack inlet opening, $\ell=\gamma \delta$, with $\gamma$ a dimensionless constant. The solvent pressure within the crack, which is approximately uniform and equal to the pressure $p$ at the inlet [13], exerts a load on the brittle solid. Thus, both $p$ and the applied far-field stress $\sigma_{\infty}$ determine the stress field in the crack region, which controls crack geometry and propagation. Then, according to linear elastic fracture mechanics, the asymptotic stress field for an edge crack of length $a$ in polar coordinates $(r, \theta)$ centered at the crack tip is [15]

$\boldsymbol{\sigma}(r, \theta) \sim \frac{K_{\mathrm{I}}}{\sqrt{2 \pi r}} \mathbf{f}(\theta), \quad K_{\mathrm{I}}=\alpha\left(\sigma_{\infty}+p\right) \sqrt{\pi a}$,

where $\alpha \approx 1.12, \mathbf{f}(\theta)$ is a dimensionless function, and $K_{\mathrm{I}}$ is the mode I stress intensity factor. Following Griffith's fracture criterion [1], crack propagation occurs whenever $K_{\mathrm{I}}$ attains the critical value $K_{\mathrm{Ic}}=\sqrt{\bar{E} \Gamma}$ called the fracture toughness. Here, $\Gamma$ is a material property representing the energy per unit area needed to create new crack surface. In Griffith's theory, $K_{\mathrm{I}}=K_{\mathrm{Ic}}$ during crack propagation, which can thus be viewed as the equation of motion of the crack tip [1].

The volume of the crack cavity per unit depth depends on the stress state following [15]

$$
A=\frac{\omega}{\alpha} \frac{K_{\mathrm{I}}}{\bar{E}} \sqrt{\pi a^{3}}
$$

where $\omega \approx 1.26$. On the other hand, crack geometry couples to solvent hydrodynamics through mass conservation of solvent exchanged between the hydrogel and the crack

$$
\dot{A}=q=k \frac{\Delta p}{\gamma},
$$

where a superposed dot denotes the time derivative. These relations highlight the intimate coupling between fracture mechanics and solvent hydraulics in the gel: Eq. (1) shows that solvent pressure governs crack propagation; conversely, Eq. (3) shows that crack propagation necessarily involves solvent transport between the gel and the crack cavity.

To examine the coupling between gel hydraulics and fracture behavior, we subject the system to a constant strain-rate $\dot{\varepsilon}$. We distinguish two stages in the dynamics of the system: (i) crack opening or closing at fixed crack length, and (ii) crack propagation. During (i), the stress intensity factor lies below the critical threshold. Since in this regime $a=a_{0}$, Eq. (3) provides an equation for crack pressure evolution that, in the dimensionless form, reads

$$
\frac{d \tilde{p}}{d \tau}=-\frac{\dot{\varepsilon}}{|\dot{\varepsilon}|}-\tilde{k}\left(\frac{2 G}{\bar{E}} \varepsilon+\tilde{p}\right),
$$

where $\tilde{p}=p / \bar{E}, \tau=|\dot{\varepsilon}| t$, and $\tilde{k}=k \bar{E} /\left(\pi \omega \gamma a_{0}^{2}|\dot{\varepsilon}|\right)$ is the dimensionless diffusivity. See Ref. [13] for a detailed derivation. Solving Eq. (4) with initial condition $\tilde{p}(0)=0$, we obtain

$$
\Delta \tilde{p}(\tau)=\frac{\dot{\varepsilon}}{|\dot{\varepsilon}|} \frac{\zeta}{\tilde{k}}[1-\exp (-\tilde{k} \tau)],
$$

where $\zeta=1-2 G / \bar{E}$.

Equation (5) clearly shows that the sign of $\Delta \tilde{p}$, and, hence, that of $q$ determining whether the solvent enters or leaves the crack cavity, crucially depends on the sign of the strain rate (tensile $\dot{\varepsilon}>0$ or compressive $\dot{\varepsilon}<0$ ) and on 
the parameter $\zeta$ characterizing the relative stiffness of the hydrogel and of the brittle elastic material. The different scenarios resulting from this analysis of stage (i) are depicted graphically in Fig. 1(b), where the top (bottom) quadrants corresponds to a stiff (compliant) hydrogel relative to the elastic layer, and right (left) corresponds to tension (compression). We discuss first the lower quadrants $(\zeta>0)$, where the stiffness of the elastic layer dominates that of the hydrogel. Under tension, the solvent is drawn to the crack cavity, $\dot{q}(0)>0$, thereby increasing its volume and, consequently, the stress intensity factor; see Eq. (2). Thus, not surprisingly, this situation eventually leads to elastic fracture under tension $(E F+)$. In this scenario, the negative pressure in the gel and in the crack cavity are not able to outweigh the positive tensile stress in Eq. (1). Under compression, any solvent within the crack is drawn to the gel $[\dot{q}(0)<0]$ and compressive stresses in the elastic layer arrest crack propagation $(E A-)$. The situation becomes more interesting in the upper quadrants, where the elastic layer is very compliant $(\zeta<0)$. Now under compression, the positive pressure within the crack cavity is able to overcome the negative stress in the brittle layer, resulting in $\dot{q}(0)>0$, increase of crack volume and $K_{\mathrm{I}}$, and eventually hydraulic fracturing $(H F-)$. Thus, the model provides a simple rationalization of the unexpected behavior reported in epithelial monolayers after stretch cessation mentioned earlier [4]. Equally counterintuitive is the tensile scenario with $\zeta<0$, where the negative pressure in the crack cavity outweighs the tensile stress, leading to crack closure and hydraulic arrest of fracture $(H A+)$; see movie 1 in the Supplemental Material [13]. Thus, when bonded to a hydrogel, cracks can open under compression and the crack opening can be resisted hydraulically under tension.

In scenarios $E F+$ and $H F-$, as the crack opens $[\dot{q}(0)>0]$ in stage (i), the pressure in the crack $p=$ $-2 G \varepsilon-\Delta p$ evolves according to Eq. (5), leading to an increase in the stress intensity factor [Eq. (1)], which eventually meets the propagation criterion at time $t^{\star}$, $K_{\mathrm{I}}\left(t^{\star}\right)=K_{\mathrm{Ic}}$. Hereafter, the system enters stage (ii) and the crack grows. The condition $K_{\mathrm{I}}(t)=K_{\mathrm{Ic}}$, together with Eqs. (1)-(3), leads to the following differential equation governing crack length [13]:

$$
\frac{d \tilde{a}}{d \tau}=\frac{2 \tilde{k}}{3 \sqrt{\tilde{a}}}\left[\frac{\zeta}{\tilde{K}_{\mathrm{Ic}}} \varepsilon-\frac{1}{\sqrt{\tilde{a}}}\right]
$$

where $\tilde{a}=a / a_{0}$ and $\tilde{K}_{\mathrm{Ic}}=K_{\mathrm{Ic}} /\left(\alpha \bar{E} \sqrt{\pi a_{0}}\right)$.

Importantly, Eq. (6) shows that the crack tip velocity $a_{0}|\dot{\varepsilon}| d \tilde{a} / d \tau$ is finite and proportional to the solvent diffusivity in the hydrogel. In contrast, without the poroelastic coupling, the condition $K_{\mathrm{I}}(t)=K_{\mathrm{Ic}}$ is no longer possible for applied strains beyond a given threshold [Eq. (1)] and quasistatic crack propagation becomes unstable $[13,16]$. The conventional way to explain the fracture physics beyond $t^{\star}$ is to invoke inertial forces [1]. Here, instead, the poroelastic coupling provides a stabilizing mechanism and governs well-posed crack dynamics.

We perform calculations of stages (i) and (ii) in the crack propagation scenarios $(E F+$ and $H F-)$ with the model above, integrating Eq. (6) numerically. Additionally, we consider a detailed fully nonlinear model, coupling large deformations of a neo-Hookean elastic solid with cohesive surfaces along predefined vertical paths to model interfacial fracture mechanics [1], a finite deformation model for the mechanics and fluid transport in the hydrogel $[12,17]$, and a one-dimensional hydrodynamic model for flow within the crack invoking lubrication theory $[13,18,19]$. This model, which considers elastic and hydrogel layers of finite thickness, is numerically approximated with the finite element method. Strikingly, we find excellent, almost quantitative, agreement between the simple mathematical model and the detailed numerical calculations [13]. Figure 2 shows representative simulations of tensile cracking (a),(b) and compressive hydraulic fracturing (c),(d) for several solvent diffusivities; see also movies 2 and 3 in the Supplemental Material [13]. For tensile cracking, during stage (i) the pressure is negative and its magnitude increases with time, thereby delaying the onset of fracture [Eq. (1)]. However, because $\zeta>0$, the hydraulic pressure is not sufficient to counterbalance the stress $\sigma_{\infty}$ and the system enters stage (ii). Then, the solvent flux, pressure magnitude, and crack length rapidly increase with time. As predicted theoretically, the crack speed increases with solvent diffusivity. For compressive hydraulic fracturing, Figs. 2(c) and 2(d), the pressure in the crack cavity
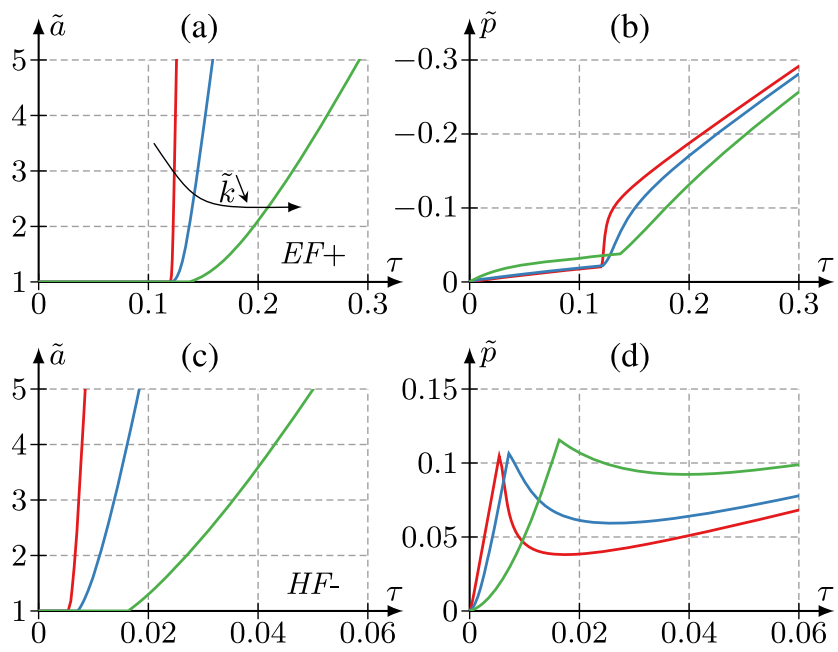

FIG. 2 (color online). Opening and propagation dynamics of a single crack for different solvent diffusivities. Tensile cracking $(E F+)$ is shown in (a),(b), $\zeta=0.8$, and compressive hydraulic fracturing $(H F-)$ is shown in (c), (d), $\zeta=-19$. The plots depict crack length $\tilde{a}$ and pressure $\tilde{p}$ in the crack cavity computed by solving Eqs. (4) and (6), with $\tilde{K}_{\text {Ic }} \approx 0.1$ and three different dimensionless diffusivities $\tilde{k} \approx 5500$ (red), 550 (blue), and 55 (green). 
increases sharply in stage (i), and is then partially and transiently released in the initial phase of stage (ii) by crack propagation and the subsequent increase of volume of the crack cavity. At later times, however, pressure increases again. The figure shows that solvent diffusivity not only controls crack propagation speed, but also its onset. Thus, the hydrogel nature of the material bonded to the brittle layer, in particular the resistance to solvent motion, delays and slows down the propagation of cracks.

Brittle materials are highly sensitive to preexisting flaws and typically fail catastrophically, by localizing deformation through growth of a single crack. For instance, in an elastic brittle layer with two parallel cracks under tension, the slightest asymmetry will cause only one of the cracks to propagate dynamically [20]. In contrast, during the hydraulic fracturing observed in epithelia, nearly all cell-cell junctions exhibited fracture [4]. Since cell-cell separation requires a significant amount of work, this distributed cracking mechanism maximizes energy dissipation before failure (toughness). Interestingly, a variety of natural materials, such as nacre, enamel, or silk, exhibit enhanced toughness by distributed cracking thanks to diverse material architectures [8-10]. We have already seen that the poroelastic coupling can arrest tensile cracking $(H A+)$ and thus make the brittle layer flaw tolerant. We examine next whether solvent hydraulics can also explain the observed distributed cracking.

As a prototype problem, we consider a brittle elastic layer with two small precracks of length $a_{0}$ bonded to a hydrogel layer, and subject the system to constant strain rate. We introduce an asymmetry by making the right crack weaker, $\Gamma_{R}=0.9 \Gamma_{L}$. Figure 3 shows the numerical results for $E F+($ a),(b) and for $H F-$ (c),(d) considering several solvent diffusivities, see also movie 4 in Ref. [13]. As a measure of the asymmetry during crack propagation, we plot the relative length difference $\Delta \tilde{a}=\left(a_{L}-a_{R}\right) / a_{0}$ as a function of time. We also report snapshots of the system immediately prior to failure, along with the strain at failure $\varepsilon_{f}$ and the normalized work of fracture at failure $\tilde{W}_{f}=\left[\Gamma_{L}\left(a_{L}-a_{0}\right)+\Gamma_{R}\left(a_{R}-a_{0}\right)\right] /\left(\Gamma_{L}+\Gamma_{R}\right) a_{0}$ as a measure of toughness. Here, failure is interpreted as the formation of a channel across the impermeable layer susceptible of cracking. It can be observed that for high diffusivity, the behavior is similar to that in which the hydrogel is absent or the bottom material is not a hydrogel, with a single crack propagating. In sharp contrast, as the diffusivity decreases both cracks advance almost equally despite the $10 \%$ weakening of the right crack. Thus, although propagating just one of the cracks is energetically favorable, the mechanism of simultaneously propagating cracks is kinetically selected for small $\tilde{k}$. This results in increased work of fracture and strain at failure by approximately twofold. We note that this behavior is different from that observed in certain sedimentary rocks and laminated engineering materials, where multiple cracks develop sequentially [21,22].

Despite its simplicity and lack of biological specificity, our model captures a number of experimental observations from Ref. [4] regarding compressive hydraulic fracturing. These experiments first considered gels of $G=4 \mathrm{kPa}$ and suddenly released stretch $(\dot{\varepsilon}<0$ and $\tilde{k}$ large). Taking $\bar{E} \approx$ $1 \mathrm{kPa}$ for cells [23], this results in $\zeta<0$. In agreement with our predictions, distributed fracture was observed at nearly

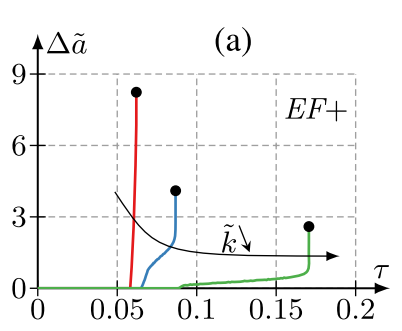

(c)

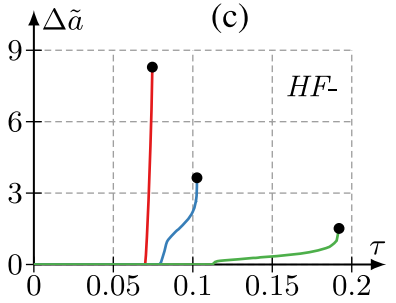

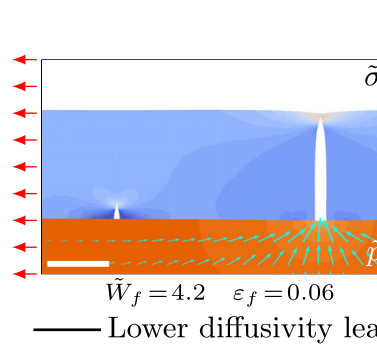

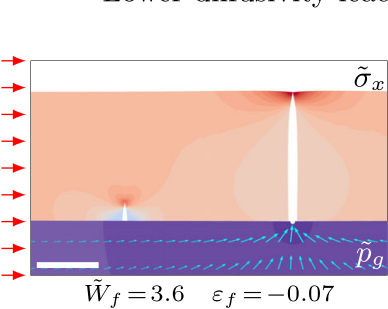

(b)

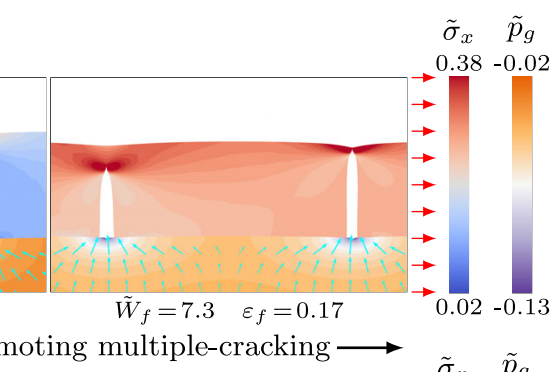

(d)
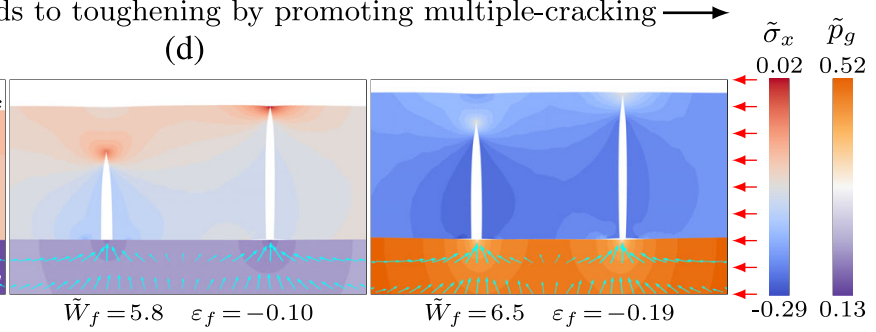

FIG. 3 (color online). Dynamics of two competing cracks for different solvent diffusivities, in tension (a),(b), $\zeta=0.8$, and in compression (c),(d), $\zeta=-1$. The plots in (a),(c) show the relative crack length difference $\Delta \tilde{a}$ for $\tilde{k} \approx 5500$ (red), 550 (blue), and 55 (green), with $|\dot{\varepsilon}|=0.01 \mathrm{~s}^{-1}$. The contour plots in (b),(d) show the longitudinal stress $\tilde{\sigma}_{x}$ in the brittle layer and the pressure $\tilde{p}_{g}$ in the hydrogel immediately prior to failure for the same values of $\tilde{k}$ as in (a),(c), decreasing from left to right. The scale bar is $5 \mu$ m; the arrows in the hydrogel represent the solvent flux. 
all cell-cell junctions. When experiments were performed with a more compliant gel $(G=0.07 \mathrm{kPa})$ for which $\zeta$ becomes positive, no cracks were observed in agreement with our model. Interestingly, freely suspended cell monolayers devoid of hydrogel matrix under stretch exhibit brittle and catastrophic fracture, propagating a single macroscopic crack [24].

In conclusion, we have shown that the hydraulic coupling between a brittle elastic solid and an adjacent poroelastic medium fundamentally modifies fracture physics. Depending on the sign of strain and the relative stiffness of the two materials, the system can exhibit unexpected behaviors, such as compressive hydraulic fracture or hydraulic arrest of cracks under tension. Furthermore, because fracture requires flow into the crack, the propagation velocity is controlled by solvent diffusion in the hydrogel. Finally, we have shown that, for low diffusivities, the bilayer system develops a toughening mechanism by promoting the simultaneous propagation of multiple cracks. Besides their relevance in epithelial integrity, our findings may have a broader biological reach since the potential cracks in epithelia, i.e., cell-cell junctions, are biologically active and the host of mechanosensitive responses [25]. In technology, our study suggests new architectures for flaw-insensitive materials with increased toughness [26-30].

A. L., G. N., and A.D.S. acknowledge the support of the European Research Council (AdG-340685MicroMotility). X. T. acknowledges the support of the European Research Council (CoG-616480), Spanish Ministry of Economy and Competitiveness (BFU201238146), and Generalitat de Catalunya (2014-SGR-927).

A. L. and G. N. contributed equally to this work.

*desimone@sissa.it

†marino.arroyo@upc.edu

[1] L. B. Freund, Dynamic Fracture Mechanics (Cambridge University Press, Cambridge, England, 1990).

[2] I. Svetlizky and J. Fineberg, Nature (London) 509, 205 (2014).

[3] B. K. Atkinson, Fracture Mechanics of Rock (Academic Press, London, 1989).

[4] L. Casares, R. Vincent, D. Zalvidea, N. Campillo, D. Navajas, M. Arroyo, and X. Trepat, Nat. Mater. 14, 343 (2015).

[5] B. Alberts, D. Bray, K. Hopkin, A. Johnson, J. Lewis, M. Raff, K. Roberts, and P. Walter, Essential Cell Biology (Garland Science, New York, 2010).

[6] Q. Saleem, B. Liu, C. C. Gradinaru, and P. M. Macdonald, Biomacromolecules 12, 2364 (2011).
[7] A. B. Imran, T. Seki, and Y. Takeoka, Polym. J. 42, 839 (2010).

[8] R. Z. Wang, Z. Suo, A. G. Evans, N. Yao, and I. A. Aksay, J. Mater. Res. 16, 2485 (2001).

[9] H. Gao, B. Ji, I. L. Jager, E. Arzt, and P. Fratzl, Proc. Natl. Acad. Sci. U.S.A. 100, 5597 (2003).

[10] S. Keten, Z. Xu, B. Ihle, and M. J. Buehler, Nat. Mater. 9, 359 (2010).

[11] A. Lucantonio, P. Nardinocchi, and H. A. Stone, J. Appl. Phys. 115, 083505 (2014).

[12] A. Lucantonio, P. Nardinocchi, and L. Teresi, J. Mech. Phys. Solids 61, 205 (2013).

[13] See Supplemental Material at http://link.aps.org/ supplemental/10.1103/PhysRevLett.115.188105, which includes Ref. [14], for additional information on the theoretical model and for videos illustrating the fracture behavior.

[14] L. Formaggia and A. Veneziani, Tech. Rep. 21, MOXPolitecnico di Milano, Milan, 2003.

[15] H. Tada, P. C. Paris, and G. R. Irwin, The Stress Analysis of Cracks Handbook (ASME Press, New York, 2000).

[16] J. Fineberg, S. P. Gross, M. Marder, and H. L. Swinney, Phys. Rev. Lett. 67, 457 (1991).

[17] W. Hong, X. Zhao, J. Zhou, and Z. Suo, J. Mech. Phys. Solids 56, 1779 (2008).

[18] T. J. Boone and A. R. Ingraffea, Int. J. Numer. Anal. Meth. Geomech. 14, 27 (1990).

[19] G. K. Batchelor, An Introduction to Fluid Dynamics (Cambridge University Press, Cambridge, England, 1967).

[20] G. Noselli, V. S. Deshpande, and N. A. Fleck, Int. J. Fract. 183, 241 (2013).

[21] T. Bai, D. D. Pollard, and H. Gao, Nature (London) 403, 753 (2000).

[22] M. Adda-Bedia and M. B. Amar, Phys. Rev. Lett. 86, 5703 (2001).

[23] J. Alcaraz, L. Buscemi, M. Grabulosa, X. Trepat, B. Fabry, R. Farré, and D. Navajas, Biophys. J. 84, 2071 (2003).

[24] A. R. Harris, L. Peter, J. Bellis, B. Baum, A. J. Kabla, and G. T. Charras, Proc. Natl. Acad. Sci. U.S.A. 109, 16449 (2012)

[25] D. J. Tschumperlin, G. Dai, I. V. Maly, T. Kikuchi, L. H. Laiho, A. K. McVittie, K. J. Haley, C. M. Lilly, P. T. C. So, D. A. Lauffenburger, R. D. Kamm, and J. M. Drazen, Nature (London) 429, 83 (2004).

[26] Z. Tang, N. A. Kotov, S. Magonov, and B. Ozturk, Nat. Mater. 2, 413 (2003).

[27] H. P. Zhang, J. Niemczura, G. Dennis, K. Ravi-Chandar, and M. Marder, Phys. Rev. Lett. 102, 245503 (2009).

[28] Z. Zhang, Y.-W. Zhang, and H. Gao, Proc. R. Soc. B 278, 519 (2011).

[29] M. Mirkhalaf, A. K. Dastjerdi, and F. Barthelat, Nat. Commun. 5 (2014).

[30] S. M. M. Valashani and F. Barthelat, Bioinsp. Biomim. 10, 026005 (2015). 